ESAIM: M2AN 50 (2016) 677-698

DOI: $10.1051 / \mathrm{m} 2 \mathrm{an} / 2015077$
ESAIM: Mathematical Modelling and Numerical Analysis

www.esaim-m2an.org

\title{
GEOMETRICALLY DEFINED BASIS FUNCTIONS FOR POLYHEDRAL ELEMENTS WITH APPLICATIONS TO COMPUTATIONAL ELECTROMAGNETICS
}

\author{
Lorenzo Codecasa $^{1}$, Ruben Specogna ${ }^{2}$ and Francesco Trevisan $^{1}$
}

\begin{abstract}
In the recent years, reformulating the mathematical description of physical laws in an algebraic form using tools from algebraic topology gained popularity in computational physics. Physical variables are defined as fluxes or circulations on oriented geometric elements of a pair of dual interlocked cell complexes, while physical laws are expressed in a metric-free fashion with incidence matrices. The metric and the material information are encoded in the discrete counterpart of the constitutive laws of materials, also referred to as constitutive or material matrices. The stability and consistency of the method is guaranteed by precise properties (symmetry, positive definiteness, consistency) that material matrices have to fulfill. The main advantage of this approach is that material matrices, even for arbitrary star-shaped polyhedral elements, can be geometrically defined, by simple closed-form expressions, in terms of the geometric elements of the primal and dual grids. That is why this original technique may be considered as a "Discrete Geometric Approach" (DGA) to computational physics. This paper first details the set of vector basis functions associated with the edges and faces of a polyhedral primal grid or of a dual grid. Then, it extends the construction of constitutive matrices for bianisotropic media.
\end{abstract}

Mathematics Subject Classification. 65N06, 65N30, 78-08.

Received March 30, 2015. Revised July 22, 2015.

Published online May 23, 2016.

\section{INTRODUCTION}

In the recent years, methods alternative to the widely used Finite Elements started to thrive and develop within the computational electromagnetics community. We may mention Yee [32] with the Finite Differences in Time Domain (FDTD) method, Weiland [13] with the Finite Integration Technique (FIT), Tonti [31] with the Cell Method (CM), Bossavit [6,8] with a reinterpretation of Finite Element Method (FEM) and the present Authors $[16,17,20]$ with the Discrete Geometric Approach (DGA).

This paper focuses on DGA, which puts emphasis on the geometric structure hidden behind Maxwell's equations. First, physical variables are defined as fluxes or circulations on oriented geometric elements of a pair of dual interlocked cell complexes. Second, physical laws are expressed in a metric-free fashion with incidence

\footnotetext{
Keywords and phrases. Discrete Geometric Approach (DGA), discrete constitutive equations, discrete hodge star operator, non-orthogonal polyhedral dual grids, bianisotropic media.

1 Politecnico di Milano, Piazza Leonardo da Vinci 32, 20133 Milan, Italy.

2 Università di Udine, Via delle Scienze 206, 33100 Udine, Italy. ruben.specogna@uniud.it
} 
matrices. These sparse matrices contain integers that describe how the geometric elements of the grid are interconnected together. Third, the metric and the material information are encoded in the discrete counterpart of the constitutive laws of materials only. This discrete counterpart of material laws, also referred to as constitutive matrices or discrete Hodge operators [30], have to fulfill precise properties (symmetry, positive definiteness, consistency) to guarantee the stability and consistency of the method $[9,27]$. The stability enforces the constitutive matrices to be symmetric and positive definite. The consistency enforces that constitutive matrices exactly map circulations onto fluxes or vice versa, at least for element-wise uniform fields.

Stable and consistent discrete constitutive equations can be constructed very easily for pairs of orthogonal Cartesian grids as shown by Yee [32] and Weiland [13]. Besides, as shown by Bossavit [7], the mass matrices constructed by means of edge and face elements introduced by Whitney and generalized by Nedelec [28, 29], satisfy both the stability and consistency properties required by DGA [7], for pairs of grids in which the primal is composed of tetrahedra and the dual grid is obtained according to the barycentric subdivision of the primal. Unfortunately, this result does not hold in general for edge and face elements relative to different geometries. For example, the present Authors have proven in [18] that Whitney's elements for generic hexahedral primal grids do not satisfy the consistency property required by DGA, for any choice of the dual grid.

The novelty content of this paper is to deduce, in greater details than [20], four general sets of vector functions for polyhedral primal grids associated with edges and faces of both the primal and of the dual grids. They are constructed directly in terms of the geometric elements (edges and faces) of the primal and of the dual grids.

We remark that the DGA, CM and FIT methods have been recently reinterpreted, within a unified framework, as compatible discrete operator schemes $[4,5]$. We also mention that our face basis functions only are akin to the same functions presented in Hybrid Finite Volume (HFV) scheme [23].

We note that some other approaches can be found in literature for generating discrete counterparts of constitutive relations over polyhedral grids, such that the mimetic finite differences $[10,12]$, the FIT [22] and the mixed finite elements [25]. However, all of these methods do not lead in general to discrete constitutive relations satisfying the consistency property required by DGA. A further result of this paper is to extend the construction of constitutive matrices to bianisotropic media [26].

Numerical experiments demonstrate that the novel discrete constitutive matrices can be computed easily and very efficiently leading to accurate approximations of the solution of both a magnetostatic and full wave electromagnetics problem proposed as a benchmark problem.

\section{DuAl INTERLOCKED GRIDS AND THEIR GEOMETRIC PROPERTIES}

Without losing generality, we focus on a primal grid consisting of a single star-shaped polyhedron $v$, Figure 1. The geometric elements of the primal grid are nodes, edges, faces and the volume $v$. We denote a primal edge with $e_{i}$, where $i=1, \ldots, L, L$ being the number of edges of $v$ and a primal face with $f_{j}$, where $j=1, \ldots, F, F$ being the number of faces of $v$. The geometric elements of the primal grid as $e_{i}, f_{j}$ are endowed with an inner orientation [31]. For example in Figure 1 the arrows indicate a possible choice of inner orientations for edge $e_{i}$ and face $f_{j}$ respectively.

A dual grid, interlocked with the primal grid, is also introduced. Each geometric element of the dual grid is in a one-to-one correspondence (i.e. dual) with a geometric element of the primal grid. The dual of a primal volume $v$ is the dual node denoted as $\tilde{v}$, where symbol " " acts on the geometric entity yielding its corresponding dual. Similarly, the dual of a primal edge $e_{i}$ is the dual face $\tilde{e}_{i}$ and the dual of a primal face $f_{j}$ is the dual edge $\tilde{f}_{j}$, Figure 1 .

\subsection{Dual grid construction}

Contrary to the usual barycentric subdivision of a simplicial primal grid to construct the dual grid, in this paper we consider a different recipe. We construct the dual grid by first introducing a dual node $\tilde{v}$ located arbitrarily within $v$. Then, the geometric construction of dual edges and dual faces is based on the barycentric subdivision of the boundary of $v$ as follows. We introduce the barycenters $g_{e_{i}}, g_{f_{j}}$ of $e_{i}, f_{j}$ respectively. 


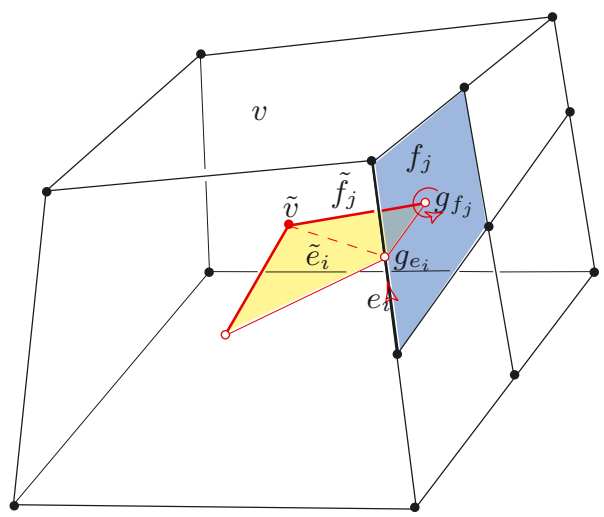

Figure 1. A polyhedron $v$. Primal edge $e_{i}$ and the dual face $\tilde{e}_{i}$. Primal face $f_{j}$ and dual edge $\tilde{f}_{j}$.

The barycenters $g_{e_{i}}$ and $g_{f_{j}}$ of edge $e_{i}$ and of face $f_{j}$ are also shown.

A dual edge $\tilde{f}_{j}$ is a segment whose boundary are the nodes $\tilde{v}, g_{f_{j}}$. A dual face $\tilde{e}_{i}$ is a quadrilateral surface made of the union of a pair of triangles; one of the triangles has the nodes $\tilde{v}, g_{e_{i}}, g_{f_{j}}$ as vertices, similarly for the other, Figure 1 . The only geometric hypothesis on $v$ we assume is that all faces $\tilde{e}_{i}$ and edges $\tilde{f}_{j}$ are contained in $v$. It is straightforward to verify that a sufficient, but not a necessary, condition is the convexity of $v$ [15].

Dual edge $\tilde{f}_{j}$ and dual face $\tilde{e}_{i}$ are endowed with outer orientation $[9,31]$, in such a way that the pairs $\left(e_{i}, \tilde{e}_{i}\right)$, $\left(f_{j}, \tilde{f}_{j}\right)$ are oriented in a congruent way.

\subsection{Geometric properties}

Vector $\mathrm{e}_{i}$, denoted in roman type, is the edge vector ${ }^{3}$ associated with the edge $e_{i}$. Vector $\mathrm{f}_{j}$ is the face vector associated with the face $f_{j}$ defined as $\mathrm{f}_{i}=\int_{f_{j}} \mathrm{nd} s$, where $\mathrm{n}$ is the unit vector normal to and oriented as $f_{j}$. Similarly vector $\tilde{\mathrm{e}}_{i}$ is the face vector associated with dual face $\tilde{e}_{i}$ and $\tilde{\mathrm{f}}_{j}$ is the edge vector associated with dual edge $\tilde{f}_{j}$.

We denote with

$$
\mathrm{T}_{i}^{e}=\tilde{\mathrm{e}}_{i} \otimes \mathrm{e}_{i}
$$

the double tensor $\mathrm{T}_{i}^{e}$ obtained from the tensor product $\otimes$ of the two vectors $\mathrm{e}_{i}$ and its dual $\tilde{\mathrm{e}}_{i}$, with $i=1, \ldots, L$. Its Cartesian components are $\left(\mathrm{T}_{i}^{e}\right)_{h k}=\left(\mathrm{e}_{i} \otimes \tilde{\mathrm{e}}_{i}\right)_{h k}=\left(\mathrm{e}_{i}\right)_{h}\left(\tilde{\mathrm{e}}_{i}\right)_{k}$, where $\left(\mathrm{e}_{i}\right)_{h}$ is the $h$ th Cartesian component of $\mathrm{e}_{i}$, with $h, k=1, \ldots, 3$. The trace of $\mathrm{T}_{i}^{e}$ is

$$
t_{i}^{e}=\operatorname{tr}\left(\mathrm{T}_{i}^{e}\right)=\mathrm{e}_{i} \cdot \tilde{\mathrm{e}}_{i}
$$

where "." is the usual inner product between vectors. The product $\mathrm{T}_{i}^{e} \mathrm{x}$ between the double tensor $\mathrm{T}_{i}^{e}$ and a generic vector $\mathrm{x}$ is a vector and

$$
\mathrm{T}_{i}^{e} \mathrm{x}=\left(\mathrm{e}_{i} \cdot \mathrm{x}\right) \tilde{\mathrm{e}}_{i} .
$$

The identity tensor is denoted by I and $\mathrm{Ix}=\mathrm{x}$ holds. Analogous relations hold by introducing

$$
\mathrm{T}_{i}^{\tilde{e}}=\mathrm{e}_{i} \otimes \tilde{\mathrm{e}}_{i}
$$

for which

$$
\begin{gathered}
\mathrm{T}_{i}^{\tilde{e} \mathrm{x}}=\left(\tilde{\mathrm{e}}_{i} \cdot \mathrm{x}\right) \mathrm{e}_{i}, \\
t_{i}^{\tilde{e}}=\operatorname{tr}\left(\mathrm{T}_{i}^{\tilde{e}}\right)=\tilde{\mathrm{e}}_{i} \cdot \mathrm{e}_{i}
\end{gathered}
$$

\footnotetext{
${ }^{3}$ Its amplitude, direction and orientation coincide with the length, direction and orientation of $e_{i}$ respectively.
} 
with $i=1, \ldots, L$. Also

$$
\mathrm{T}_{i}^{f}=\tilde{\mathrm{f}}_{i} \otimes \mathrm{f}_{i}
$$

for which

$$
\begin{aligned}
& \mathrm{T}_{i}^{f} \mathrm{x}=\left(\mathrm{f}_{i} \cdot \mathrm{x}\right) \tilde{\mathrm{f}}_{i}, \\
& t_{i}^{f}=\operatorname{tr}\left(\mathrm{T}_{i}^{f}\right)=\mathrm{f}_{i} \cdot \tilde{\mathrm{f}}_{i}
\end{aligned}
$$

with $i=1, \ldots, F$ and

$$
\mathrm{T}_{i}^{\tilde{f}}=\mathrm{f}_{i} \otimes \tilde{\mathrm{f}}_{i}
$$

for which

$$
\begin{aligned}
& \mathrm{T}_{i}^{\tilde{f} \mathrm{x}}=\left(\tilde{\mathrm{f}}_{i} \cdot \mathrm{x}\right) \mathrm{f}_{i}, \\
& t_{i}^{\tilde{f}}=\operatorname{tr}\left(\mathrm{T}_{i}^{\tilde{f}}\right)=\tilde{\mathrm{f}}_{i} \cdot \mathrm{f}_{i}
\end{aligned}
$$

with $i=1, \ldots, F$.

Provided that the dual grid is constructed according to Section 2.1, in papers $[15,18]$ we proved the following geometric identities

$$
\sum_{i=1}^{L} \mathrm{e}_{i} \otimes \tilde{\mathrm{e}}_{i}=|v| \mathrm{I}, \quad \sum_{i=1}^{F} \mathrm{f}_{j} \otimes \tilde{\mathrm{f}}_{j}=|v| \mathrm{I},
$$

where $|v|$ is the volume of $v$. Identities (2.13) can now be conveniently rewritten as

$$
\begin{aligned}
& \mathrm{T}^{e}=\sum_{i=1}^{L} \mathrm{~T}_{i}^{e}=\sum_{i=1}^{L} \tilde{\mathrm{e}}_{i} \otimes \mathrm{e}_{i}=|v| \mathrm{I}, \\
& \mathrm{T}^{\tilde{e}}=\sum_{i=1}^{L} \mathrm{~T}_{i}^{\tilde{e}}=\sum_{i=1}^{L} \mathrm{e}_{i} \otimes \tilde{\mathrm{e}}_{i}=|v| \mathrm{I}, \\
& \mathrm{T}^{f}=\sum_{i=1}^{F} \mathrm{~T}_{i}^{f}=\sum_{i=1}^{F} \tilde{\mathrm{f}}_{i} \otimes \mathrm{f}_{i}=|v| \mathrm{I}, \\
& \mathrm{T}^{\tilde{f}}=\sum_{i=1}^{F} \mathrm{~T}_{i}^{\tilde{f}}=\sum_{i=1}^{F} \mathrm{f}_{i} \otimes \tilde{\mathrm{f}}_{i}=|v| \mathrm{I} .
\end{aligned}
$$

Obviously, tensors $\mathrm{T}^{e}, \mathrm{~T}^{\tilde{e}}, \mathrm{~T}^{f}, \mathrm{~T}^{\tilde{f}}$ are symmetric and their traces are $3|v|$.

\subsection{Partition of the polyhedron}

We introduce a partition of the polyhedron $v$ into a number of subregions $\tau_{i}^{r}$ in a one-to-one correspondence with the geometric element $e_{i}$ and $\tilde{e}_{i}$, with $i=1, \ldots, L$. Precisely, subregion $\tau_{i}^{e}=\tau_{i}^{\tilde{e}}$ is shown in Figure $2 \mathrm{~A}$, with $i=1, \ldots, L$; it is a polyhedral region individuated by $e_{i}$, or equivalently $\tilde{e}_{i}$, formed by a pair of tetrahedra, each of which having as vertices the dual node $\tilde{v}$, the pair of nodes bounding $e_{i}$, and one of the barycenters of the two primal faces having $e_{i}$ in common.

Lemma 2.1. We have that

$$
t_{i}^{e}=t_{i}^{\tilde{e}}=3\left|\tau_{i}^{e}\right|
$$

holds, where $\left|\tau_{i}^{e}\right|$ is the volume of the subregion $\tau_{i}^{e}$. 

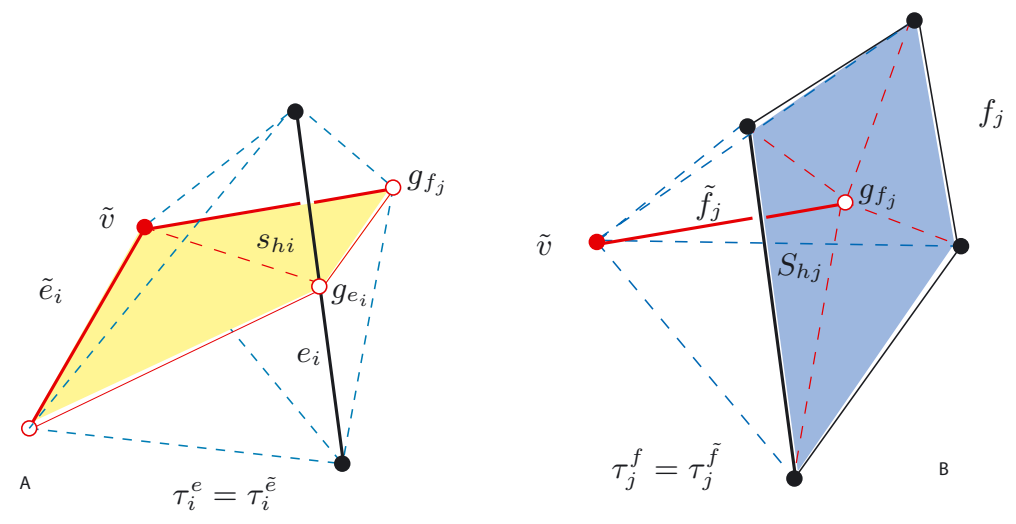

FIGURE 2. Subregions $\tau_{i}^{e}=\tau_{i}^{\tilde{e}}$ and $\tau_{j}^{f}=\tau_{j}^{\tilde{f}}$ are shown in detail.

Proof. Firstly, let us consider the case of a subregion $\tau_{i}^{e}=\tau_{i}^{\tilde{e}}$, Figure $2 \mathrm{~A}$. For the dual face $\tilde{e}_{i}$, we write $\tilde{e}_{i}=\cup_{h=1}^{2} s_{h i}$, where $s_{h i}$ is one of the two triangular portions forming $\tilde{e}_{i}$; In terms of area vectors we write $\tilde{\mathrm{e}}_{i}=\sum_{h=1}^{2} \mathrm{~S} h i$. Then, from the formula giving the volume of a tetrahedron,

$$
\mathrm{e}_{i} \cdot \tilde{\mathrm{e}}_{i}=\sum_{h=1}^{2} \mathrm{~s}_{h i} \cdot \mathrm{e}_{i}=\sum_{h=1}^{2} 3\left|V_{h}\right|=3\left|\tau_{i}^{e}\right|
$$

holds, where $\left|V_{h}\right|$ is the volume of one of the two tetrahedra $V_{h}$ forming $\tau_{i}^{e}$, Figure 3. Then from (2.2) the thesis follows.

Analogously Figure 2B, shows subregion $\tau_{j}^{f}=\tau_{j}^{\tilde{f}}$, with $j=1, \ldots, F$; it is a polyhedral region individuated by $f_{j}$ or equivalently $\tilde{f}_{j}$, formed by a pyramid having as base the $f_{j}$ face and as apex the dual node $\tilde{v}$.

Lemma 2.2. We have that

$$
t_{i}^{f}=t_{i}^{\tilde{f}}=3\left|\tau_{i}^{f}\right|
$$

holds, where $\left|\tau_{i}^{f}\right|$ is the volume of the subregion $\tau_{i}^{f}$.

Proof. Let us consider the case of a subregion $\tau_{j}^{f}=\tau_{j}^{\tilde{f}}$, Figure 2B. From the formula expressing the volume of a pyramid

$$
\mathrm{f}_{j} \cdot \tilde{\mathrm{f}}_{j}=3\left|\tau_{j}^{f}\right|
$$

holds. Then from (2.2) the thesis follows.

\section{Geometrichlly Defined VeCtor BASIS FUnCtions}

\subsection{Basis functions $v_{i}^{e}(p)$}

We consider an electric field $\mathrm{E}(p)$ in $v$; in the DGA, circulations of $\mathrm{E}(p)$ can be introduced along the primal edges

$$
E_{i}^{e}=\int_{e_{i}} \mathrm{E}(p) \cdot \mathrm{de}
$$




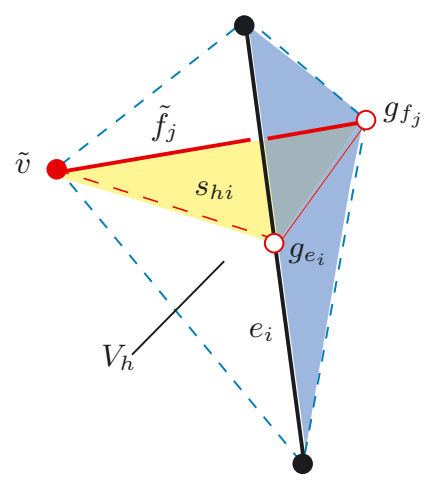

Figure 3. Detail of a tetrahedron $V_{h}$ forming the subregions $\tau_{i}^{e}=\tau_{i}^{\tilde{e}}$.

Now let us assume that $\mathrm{E}(p)$ is uniform in $v$ and thus $\mathrm{E}$ does not depend on $p$. Then by right multiplying (2.14) by E, from (2.3), (2.14) and (3.1), since $E_{i}^{e}=\mathrm{E} \cdot \mathrm{e}_{i}$, we obtain

$$
\mathrm{E}=\frac{1}{|v|} \sum_{i=1}^{L} E_{i}^{e} \tilde{\mathrm{e}}_{i}
$$

Besides, multiplying on the right by $\mathrm{x}$ both members of the identity

$$
\mathrm{I}=\frac{\mathrm{T}_{j}^{e}}{t_{j}^{e}}+\left(\mathrm{I}-\frac{\mathrm{T}_{j}^{e}}{t_{j}^{e}}\right)
$$

and using (2.3), we have that

$$
\mathrm{E}=\frac{E_{j}^{e}}{t_{j}^{e}} \tilde{\mathrm{e}}_{j}+\left(\mathrm{I}-\frac{\mathrm{T}_{j}^{e}}{t_{j}^{e}}\right) \mathrm{E}
$$

holds in subregion $\tau_{j}^{e}$, with $j=1, \ldots, L$. Thus by substituting (3.2) for $\mathrm{E}$ in the right hand side of (3.4), we obtain

$$
\mathrm{E}=\sum_{i=1}^{L}\left(\frac{\tilde{\mathrm{e}}_{j}}{t_{j}^{e}} \delta_{i j}+\left(\mathrm{I}-\frac{\mathrm{T}_{j}^{e}}{t_{j}^{e}}\right) \frac{\tilde{\mathrm{e}}_{i}}{|v|}\right) E_{i}^{e},
$$

where $\delta_{i j}$ is the Kronecker symbol, or equivalently

$$
\mathrm{e}=\sum_{i=1}^{L} \mathrm{v}_{i}^{e}(p) E_{i}^{e}
$$

in which

$$
\mathrm{v}_{i}^{e}(p)=\frac{\tilde{\mathrm{e}}_{j}}{t_{j}^{e}} \delta_{i j}+\left(\mathrm{I}-\frac{\mathrm{T}_{j}^{e}}{t_{j}^{e}}\right) \frac{\tilde{\mathrm{e}}_{i}}{|v|}, \quad \text { for each } p \in \tau_{j}^{e}, \text { with } j=1, \ldots, L .
$$

Quantities $\mathrm{v}_{i}^{e}(p)$, with $i=1, \ldots, L$, derived in this way, are vector functions, piece-wise uniform in $v$ and uniform in each subregion $\tau_{j}^{e}$ with $j=1, \ldots, L$. Moreover, they satisfy the following three properties, fundamental to construct constitutive matrices for the DGA, as outlined by the authors in $[1,14]$. 
Property 3.1. The functions $\mathrm{v}_{i}^{e}(p)$, with $i=1, \ldots, L$ are linearly independent, and are such that

$$
\int_{e_{j}} \mathrm{v}_{i}^{e}(p) \cdot \mathrm{de}=\delta_{i j}
$$

holds, for $i, j=1, \ldots, L$.

Proof. In the subregion $\tau_{j}^{e}$ adjacent to $e_{j}$, we write

$$
\begin{aligned}
\int_{e_{j}} \mathrm{v}_{i}^{e}(p) \cdot \mathrm{de} & =\left(\frac{\tilde{\mathrm{e}}_{j}}{t_{j}^{e}} \delta_{i j}+\left(\mathrm{I}-\frac{\mathrm{T}_{j}^{e}}{t_{j}^{e}}\right) \frac{\tilde{\mathrm{e}}_{i}}{|v|}\right) \cdot \mathrm{e}_{j} \\
& =\delta_{i j}+\left(\frac{\tilde{\mathrm{e}}_{i}}{|v|}-\frac{\mathrm{e}_{j} \cdot \tilde{\mathrm{e}}_{i}}{t_{j}^{e}|v|} \tilde{\mathrm{e}}_{j}\right) \cdot \mathrm{e}_{j} \\
& =\delta_{i j}+\left(\frac{\mathrm{e}_{j} \cdot \tilde{\mathrm{e}}_{i}}{|v|}-\frac{\mathrm{e}_{j} \cdot \tilde{\mathrm{e}}_{i}}{|v|}\right)=\delta_{i j}
\end{aligned}
$$

In the second equality (2.3) has been applied, while in the first and last equalities we used (2.2). Thus (3.8) holds. As a consequence functions $\mathrm{v}_{i}^{e}(p)$, with $i=1, \ldots, L$ are linearly independent.

Property 3.2. The functions $\mathrm{v}_{i}^{e}(p)$, with $i=1, \ldots, L$ allow to represent exactly a uniform vector field from its Degrees of Freedom, according to (3.6).

Proof. The thesis straightforwardly follows from (3.5).

Property 3.3. The geometric consistency condition [8]

$$
\int_{v} \mathrm{v}_{i}^{e}(p) \mathrm{d} v=\tilde{\mathrm{e}}_{i}
$$

holds, with $i=1, \ldots, L$.

Proof. We compute

$$
\begin{aligned}
\int_{v} \mathrm{v}_{i}^{e}(p) \mathrm{d} v & =\sum_{j=1}^{L} \int_{\tau_{j}^{e}} \mathrm{v}_{i}^{e}(p) \mathrm{d} v \\
& =\sum_{j=1}^{L}\left(\frac{\tilde{\mathrm{e}}_{j}}{t_{j}^{e}} \delta_{i j}+\left(\mathrm{I}-\frac{\mathrm{T}_{j}^{e}}{t_{j}^{e}}\right) \frac{\tilde{\mathrm{e}}_{i}}{|v|}\right)\left|\tau_{j}^{e}\right| \\
& =\sum_{j=1}^{L} \tilde{\mathrm{e}}_{j} \frac{\left|\tau_{j}^{e}\right|}{t_{j}^{e}} \delta_{i j}+\left(\sum_{j=1}^{L}\left|\tau_{j}^{e}\right|\right) \frac{\tilde{\mathrm{e}}_{i}}{|v|}-\frac{1}{3}\left(\sum_{j=1}^{L} \mathrm{~T}_{j}^{e}\right) \frac{\tilde{\mathrm{e}}_{i}}{|v|} \\
& =\frac{1}{3} \tilde{\mathrm{e}}_{i}+\tilde{\mathrm{e}}_{i}-\frac{1}{3} \tilde{\mathrm{e}}_{i}=\tilde{\mathrm{e}}_{i},
\end{aligned}
$$

where, in the second equality we used Lemma 2.2 and in the third equality we used the identity (2.14). 


\subsection{Basis functions $v_{i}^{f}(p)$}

We consider a magnetic induction $\mathrm{B}(p)$ in $v$; in the DGA, fluxes of $\mathrm{B}(p)$ can be introduced through the primal faces

$$
B_{i}^{f}=\int_{f_{i}} \mathrm{~B}(p) \cdot \mathrm{df}
$$

Now let us assume that $\mathrm{B}(p)$ is uniform in $v$ and thus $\mathrm{B}$ does not depend on $p$. Then by right multiplying (2.14) by $\mathrm{B}$, from $(2.8),(2.14)$ and $(3.10)$, since $B_{i}^{f}=\mathrm{B} \cdot \mathrm{f}_{i}$, we obtain

$$
\mathrm{B}=\frac{1}{|v|} \sum_{j=1}^{F} \Phi_{i}^{f} \tilde{\mathrm{f}}_{i}
$$

Besides, multiplying on the right by B both members of the identity

$$
\mathrm{I}=\frac{\mathrm{T}_{j}^{f}}{t_{j}^{f}}+\left(\mathrm{I}-\frac{\mathrm{T}_{j}^{f}}{t_{j}^{f}}\right)
$$

and using (2.8), we have that

$$
\mathrm{B}=\frac{B_{j}^{f}}{t_{j}^{f}} \tilde{\mathrm{f}}_{j}+\left(\mathrm{I}-\frac{\mathrm{T}_{j}^{f}}{t_{j}^{f}}\right) \mathrm{B}
$$

holds in subregion $\tau_{j}^{f}$, with $j=1, \ldots, F$. Thus by substituting (3.11) for B in the right hand side of (3.13), we obtain

$$
\mathrm{B}=\sum_{i=1}^{L}\left(\frac{\tilde{\mathrm{f}}_{j}}{t_{j}^{f}} \delta_{i j}+\left(\mathrm{I}-\frac{\mathrm{T}_{j}^{f}}{t_{j}^{f}}\right) \frac{\tilde{\mathrm{f}}_{i}}{|v|}\right) B_{i}^{f},
$$

where $\delta_{i j}$ is the Kronecker symbol, or equivalently

$$
\mathrm{B}=\sum_{i=1}^{F} \mathrm{v}_{i}^{f}(p) B_{i}^{f}
$$

in which

$$
\mathrm{v}_{i}^{f}(p)=\frac{\tilde{\mathrm{f}}_{j}}{t_{j}^{f}} \delta_{i j}+\left(\mathrm{I}-\frac{\mathrm{T}_{j}^{f}}{t_{j}^{f}}\right) \frac{\tilde{\mathrm{f}}_{i}}{|v|}, \quad \text { for each } p \in \tau_{j}^{f}, \text { with } j=1, \ldots, F .
$$

Quantities $\mathrm{v}_{i}^{f}(p)$, with $i=1, \ldots, F$, derived in this way, are vector functions, piece-wise uniform in $v$ and uniform in each subregion $\tau_{j}^{f}$ with $j=1, \ldots, F$. Moreover, they satisfy the following three properties, fundamental to construct constitutive matrices for the DGA, as outlined by the authors in $[1,14]$.

Property 3.4. The functions $\mathrm{v}_{i}^{f}(p)$, with $i=1, \ldots, F$ are linearly independent, and are such that

$$
\int_{f_{j}} \mathrm{v}_{i}^{f}(p) \cdot \mathrm{df}=\delta_{i j}
$$

holds, for $i, j=1, \ldots, F$. 
Proof. In the subregion $\tau_{j}^{f}$ adjacent to $f_{j}$, we write

$$
\begin{aligned}
\int_{f_{j}} \mathrm{v}_{i}^{f}(p) \cdot \mathrm{df} & =\left(\frac{\tilde{\mathrm{f}}_{j}}{t_{j}^{f}} \delta_{i j}+\left(\mathrm{I}-\frac{\mathrm{T}_{j}^{f}}{t_{j}^{f}}\right) \frac{\tilde{\mathrm{f}}_{i}}{|v|}\right) \cdot \mathrm{f}_{j} \\
& =\delta_{i j}+\left(\frac{\tilde{\mathrm{f}}_{i}}{|v|}-\frac{\mathrm{f}_{j} \cdot \tilde{\mathrm{f}}_{i}}{t_{j}^{f}|v|} \tilde{\mathrm{f}}_{j}\right) \cdot \mathrm{f}_{j} \\
& =\delta_{i j}+\left(\frac{\mathrm{f}_{j} \cdot \tilde{\mathrm{f}}_{i}}{|v|}-\frac{\mathrm{f}_{j} \cdot \tilde{\mathrm{f}}_{i}}{|v|}\right)=\delta_{i j} .
\end{aligned}
$$

In the second equality (2.8) has been applied, while in the first and last equalities we used (2.2). Thus (3.17) holds. As a consequence functions $\mathrm{v}_{i}^{f}(p)$, with $i=1, \ldots, F$ are linearly independent.

Property 3.5. The functions $\mathrm{v}_{i}^{f}(p)$, with $i=1, \ldots, F$ allow to represent exactly a uniform vector field from its Degrees of Freedom, according to (3.15).

Proof. The thesis straightforwardly follows from (3.14).

Property 3.6. The geometric consistency condition [8]

$$
\int_{v} \mathrm{v}_{i}^{f}(p) \mathrm{d} v=\tilde{\mathrm{f}}_{i}
$$

holds, with $i=1, \ldots, F$.

Proof. We compute

$$
\begin{aligned}
\int_{v} \mathrm{v}_{i}^{f}(p) \mathrm{d} v & =\sum_{j=1}^{F} \int_{\tau_{j}^{f}} \mathrm{v}_{i}^{f}(p) \mathrm{d} v \\
& =\sum_{j=1}^{F}\left(\frac{\tilde{\mathrm{f}}_{j}}{t_{j}^{f}} \delta_{i j}+\left(\mathrm{I}-\frac{\mathrm{T}_{j}^{f}}{t_{j}^{f}}\right) \frac{\tilde{\mathrm{f}}_{i}}{|v|}\right)\left|\tau_{j}^{f}\right| \\
& =\sum_{j=1}^{F} \tilde{\mathrm{f}}_{j} \frac{\left|\tau_{j}^{f}\right|}{t_{j}^{f}} \delta_{i j}+\left(\sum_{j=1}^{F}\left|\tau_{j}^{f}\right|\right) \frac{\tilde{\mathrm{f}}_{i}}{|v|}-\frac{1}{3}\left(\sum_{j=1}^{L} \mathrm{~T}_{j}^{f}\right) \frac{\tilde{\mathrm{f}}_{i}}{|v|} \\
& =\frac{1}{3} \tilde{\mathrm{f}}_{i}+\tilde{\mathrm{f}}_{i}-\frac{1}{3} \tilde{\mathrm{f}}_{i}=\tilde{\mathrm{f}}_{i},
\end{aligned}
$$

where, in the second equality we used Lemma 2.2 and in the third equality we used the identity (2.14).

\subsection{Basis functions $v_{i}^{\tilde{e}}(p)$}

We consider an electric field $\mathrm{D}(p)$ in $v$; in the DGA, fluxes of $\mathrm{D}(p)$ can be introduced through the dual edges

$$
D_{i}^{\tilde{e}}=\int_{\tilde{e}_{i}} \mathrm{D}(p) \cdot \mathrm{dẽ} .
$$

Now let us assume that $\mathrm{D}(p)$ is uniform in $v$ and thus $\mathrm{D}$ does not depend on $p$. Then by right multiplying (2.14) by D, from $(2.5),(2.14)$ and $(3.19)$, since $D_{i}^{\tilde{e}}=\mathrm{D} \cdot \tilde{\mathrm{e}}_{i}$, we obtain

$$
\mathrm{D}=\frac{1}{|v|} \sum_{i=1}^{L} D_{i}^{\tilde{e}} \tilde{e}_{i} .
$$


Besides, multiplying on the right by D both members of the identity

$$
\mathrm{I}=\frac{\mathrm{T}_{j}^{\tilde{e}}}{t_{j}^{\tilde{e}}}+\left(\mathrm{I}-\frac{\mathrm{T}_{j}^{\tilde{e}}}{t_{j}^{\tilde{e}}}\right)
$$

and using (2.8), we have that

$$
\mathrm{D}=\frac{D_{j}^{\tilde{e}}}{t_{j}^{\tilde{e}}} \tilde{e}_{j}+\left(\mathrm{I}-\frac{\mathrm{T}_{j}^{\tilde{e}}}{t_{j}^{\tilde{e}}}\right) \mathrm{D}
$$

holds in subregion $\tau_{j}^{\tilde{e}}$, with $j=1, \ldots, L$. Thus by substituting (3.20) for $\mathrm{D}$ in the right hand side of (3.22), we obtain

$$
\mathrm{D}=\sum_{i=1}^{L}\left(\frac{\tilde{e}_{j}}{t_{j}^{\tilde{e}}} \delta_{i j}+\left(\mathrm{I}-\frac{\mathrm{T}_{j}^{\tilde{e}}}{t_{j}^{\tilde{e}}}\right) \frac{\mathrm{e}_{i}}{|v|}\right) D_{i}^{\tilde{e}},
$$

where $\delta_{i j}$ is the Kronecker's symbol, or equivalently

$$
\mathrm{D}=\sum_{i=1}^{L} \mathrm{v}_{i}^{\tilde{e}}(p) D_{i}^{\tilde{e}}
$$

in which

$$
\mathrm{v}_{i}^{\tilde{e}}(p)=\frac{\mathrm{e}_{j}}{t_{j}^{\tilde{e}}} \delta_{i j}+\left(\mathrm{I}-\frac{\mathrm{T}_{j}^{\tilde{e}}}{t_{j}^{\tilde{e}}}\right) \frac{\mathrm{e}_{i}}{|v|}, \quad \text { for each } p \in \tau_{j}^{\tilde{e}}, \text { with } j=1, \ldots, L .
$$

Quantities $\mathrm{v}_{i}^{\tilde{e}}(p)$, with $i=1, \ldots, L$, derived in this way, are vector functions, piece-wise uniform in $v$ and uniform in each subregion $\tau_{j}^{\tilde{e}}$ with $j=1, \ldots, L$. Moreover, they satisfy the following three properties, fundamental to construct constitutive matrices for the DGA, as outlined by the authors in [1,14]. The proofs of the properties will be omitted being similar to those reported in Section 3.1.

Property 3.7. The functions $\mathrm{v}_{i}^{\tilde{e}}(p)$, with $i=1, \ldots, L$ are linearly independent, and are such that

$$
\int_{\tilde{e}_{j}} \mathrm{v}_{i}^{\tilde{e}}(p) \cdot \mathrm{d} \tilde{\mathrm{e}}=\delta_{i j}
$$

holds, for $i, j=1, \ldots, L$.

Property 3.8. The functions $\mathrm{v}_{i}^{\tilde{e}}(p)$, with $i=1, \ldots, L$ allow to represent exactly a uniform vector field from its Degrees of Freedom, according to (3.24).

Property 3.9. The geometric consistency condition [8]

$$
\int_{v} \mathrm{v}_{i}^{\tilde{e}}(p) \mathrm{d} v=\mathrm{e}_{i}
$$

holds, with $i=1, \ldots, L$.

\subsection{Basis functions $v_{i}^{\tilde{f}}(p)$}

We consider a magnetic field $\mathrm{H}(p)$ in $v$; in the DGA, circulations of $\mathrm{H}(p)$ can be introduced along the dual edges

$$
H_{i}^{\tilde{f}}=\int_{\tilde{f}_{i}} \mathrm{H}(p) \cdot \mathrm{d} \tilde{f} .
$$


Now let us assume that $\mathrm{H}(p)$ is uniform in $v$ and thus $\mathrm{H}$ does not depend on $p$. Then by right multiplying (2.14) by $\mathrm{H}$, from $(2.11),(2.14)$ and $(3.28)$, since $H_{i}^{\tilde{f}}=\mathrm{H} \cdot \tilde{\mathrm{f}}_{i}$, we obtain

$$
\mathrm{H}=\frac{1}{|v|} \sum_{i=1}^{F} H_{i}^{\tilde{f}} \tilde{f}_{i}
$$

Besides, multiplying on the right by $\mathrm{H}$ both members of the identity

$$
\mathrm{I}=\frac{\mathrm{T}_{j}^{\tilde{f}}}{t_{j}^{\tilde{f}}}+\left(\mathrm{I}-\frac{\mathrm{T}_{j}^{\tilde{f}}}{t_{j}^{\tilde{f}}}\right)
$$

and using (2.11), we have that

$$
\mathrm{H}=\frac{H_{j}^{\tilde{f}}}{t_{j}^{\tilde{f}}} \tilde{f}_{j}+\left(\mathrm{I}-\frac{\mathrm{T}_{j}^{\tilde{f}}}{t_{j}^{\tilde{f}}}\right) \mathrm{H}
$$

holds in subregion $\tau_{j}^{\tilde{f}}$, with $j=1, \ldots, F$. Thus by substituting (3.29) for $\mathrm{H}$ in the right hand side of (3.31), we obtain

$$
\mathrm{H}=\sum_{i=1}^{F}\left(\frac{\tilde{f}_{j}}{t_{j}^{\tilde{f}}} \delta_{i j}+\left(\mathrm{I}-\frac{\mathrm{T}_{j}^{\tilde{f}}}{t_{j}^{\tilde{f}}}\right) \frac{\mathrm{f}_{i}}{|v|}\right) H_{i}^{\tilde{f}}
$$

where $\delta_{i j}$ is the Kronecker's symbol, or equivalently

$$
\mathrm{H}=\sum_{i=1}^{F} \mathrm{v}_{i}^{\tilde{f}}(p) H_{i}^{\tilde{f}}
$$

in which

$$
\mathrm{v}_{i}^{\tilde{f}}(p)=\frac{\mathrm{f}_{j}}{t_{j}^{\tilde{f}}} \delta_{i j}+\left(\mathrm{I}-\frac{\mathrm{T}_{j}^{\tilde{f}}}{t_{j}^{\tilde{f}}}\right) \frac{\mathrm{f}_{i}}{|v|}, \quad \text { for each } p \in \tau_{j}^{\tilde{f}}, \text { with } j=1, \ldots, F .
$$

Quantities $\mathrm{v}_{i}^{\tilde{f}}(p)$, with $i=1, \ldots, F$, derived in this way, are vector functions, piece-wise uniform in $v$ and uniform in each subregion $\tau_{j}^{\tilde{f}}$ with $j=1, \ldots, F$. Moreover, they satisfy the following three properties, fundamental to construct constitutive matrices for the DGA, as outlined by the authors in [1,14]. The proofs of the properties will be omitted being similar to those reported in Section 3.2.

Property 3.10. The functions $\mathrm{v}_{i}^{\tilde{f}}(p)$, with $i=1, \ldots, F$ are linearly independent, and are such that

$$
\int_{\tilde{f}_{j}} \mathrm{v}_{i}^{\tilde{f}}(p) \cdot \mathrm{d} \tilde{f}=\delta_{i j}
$$

holds, for $i, j=1, \ldots, F$.

Property 3.11. The functions $\mathrm{v}_{i}^{\tilde{f}}(p)$, with $i=1, \ldots, F$ allow to represent exactly a uniform vector field from its Degrees of Freedom, according to (3.33).

Property 3.12. The geometric consistency condition [8]

$$
\int_{v} \mathrm{v}_{i}^{\tilde{f}}(p) \mathrm{d} v=\mathrm{f}_{i}
$$

holds, with $i=1, \ldots, F$. 


\section{Constitutive matrix}

\section{1. $\mathrm{E}, \mathrm{H}$ to $\mathrm{D}, \mathrm{B}$ constitutive relations}

We consider a single polyhedron $v$, where vector fields $\mathrm{D}, \mathrm{H}, \mathrm{E}, \mathrm{B}$ exists, related by the constitutive relations

$$
\begin{aligned}
& \mathrm{D}=\boldsymbol{\varepsilon} \mathrm{E}+\boldsymbol{\xi} \mathrm{H}, \\
& \mathrm{B}=\boldsymbol{\zeta} \mathrm{E}+\boldsymbol{\mu} \mathrm{H}
\end{aligned}
$$

$\varepsilon, \boldsymbol{\xi}, \boldsymbol{\zeta}, \boldsymbol{\nu}$ being double tensors, assumed homogeneous in $v$, representing the material property. Various physical properties are satisfied by these tensors [26]. In particular, considering non-dispersive materials for the sake of simplicity, the existance of a non-negative energy implies that matrix

$$
\alpha=\left[\begin{array}{ll}
\varepsilon & \xi \\
\zeta & \mu
\end{array}\right]
$$

is symmetric positive-definite.

Now, we focus on the pairs of geometric elements $e_{i}, \tilde{e}_{i}$, one dual of the other, with $i=1, \ldots, L$ and $f_{i}$, $\tilde{f}_{i}$, one dual of the other, with $i=1, \ldots, F$ and we introduce the corresponding pair of degrees of freedom $E_{i}^{e}=\int_{e_{i}} \mathrm{E} \cdot \mathrm{de}, D_{i}^{\tilde{e}}=\int_{\tilde{e}_{i}} \mathrm{D} \cdot \mathrm{dr}$ and $B_{i}^{f}=\int_{f_{i}} \mathrm{~B} \cdot \mathrm{df}, H_{i}^{\tilde{f}}=\int_{\tilde{f}_{i}} \mathrm{H} \cdot \mathrm{df}$. We denote in boldface type the arrays $\mathbf{E}^{e}, \mathbf{D}^{\tilde{e}}$, of dimension $L$, formed by $E_{i}^{e}, D_{i}^{\tilde{e}}$ respectively, and $\mathbf{B}^{f}, \mathbf{H}^{\tilde{f}}$, of dimension $F$, formed by $B_{i}^{f}, H_{i}^{\tilde{f}}$. We introduce the discrete counterpart of (4.1) in $v$ as

$$
\begin{aligned}
& \mathbf{D}^{\tilde{e}} \cong \mathbf{M}^{e e}(\varepsilon) \mathbf{E}^{e}+\mathbf{M}^{e \tilde{f}}(\boldsymbol{\xi}) \mathbf{H}^{\tilde{f}}, \\
& \mathbf{B}^{f} \cong \mathbf{M}^{\tilde{f} e}(\boldsymbol{\zeta}) \mathbf{E}^{e}+\mathbf{M}^{\tilde{f} \tilde{f}}(\boldsymbol{\mu}) \mathbf{H}^{\tilde{f}},
\end{aligned}
$$

where $\mathbf{M}^{e e}(\varepsilon)$ is a constitutive matrix of dimension $L \times L, \mathbf{M}^{e \tilde{f}}(\boldsymbol{\xi})$ is a constitutive matrix of dimension $L \times F$, $\mathbf{M}^{\tilde{f} e}(\boldsymbol{\zeta})$ is a constitutive matrix of dimension $F \times L$, and $\mathbf{M}^{\tilde{f} \tilde{f}}(\boldsymbol{\mu})$ is a constitutive matrix of dimension $F \times F$. Equations (4.2) holds only approximately, yielding the well known constitutive error affecting the overall discrete problem [1,9]. The general case in which $\boldsymbol{\xi}=\boldsymbol{\zeta} \neq \mathbf{0}$ is typical of bianistropic electromagnetic media, while the simpler case in which $\boldsymbol{\xi}=\boldsymbol{\zeta}=\mathbf{0}$ is more common and is typical of isotropic and anisotropic electromagnetic media.

As shown in $[7,9,27]$ for anisotropic media and in [1] for bianisotropic media, the aim is to construct a constitutive matrix

$$
\mathbf{A}=\left[\begin{array}{ll}
\mathbf{M}^{e e}(\boldsymbol{\varepsilon}) & \mathbf{M}^{e \tilde{f}}(\boldsymbol{\xi}) \\
\mathbf{M}^{\tilde{f e}}(\boldsymbol{\zeta}) & \mathbf{M}^{\tilde{f} \tilde{f}}(\boldsymbol{\mu})
\end{array}\right]
$$

which complies with the following requirements: (i) it is symmetric, (ii) it is positive definite and (iii) it is such that equations (4.2) hold exactly at least for uniform fields E, H, D, B related by (4.1) in $v$. The requirements (i) and (ii) are the discrete couterpart of the symmetric, positive definite property for $\boldsymbol{\alpha}$. They are fundamental to guarantee the stability of the discretized equations while the last requirement iii) guarantees the consistency of the discretized equations in the DGA.

In order to comply with these requirements, we will resort to the so called energetic approach proposed in $[1,14,16-18]$ which relies solely on Properties 3.1.1, 3.1.2 and 3.1.3 for the vector basis functions $\mathrm{v}_{i}^{e}(p)$ with $i=1, \ldots, L$, and Properties 3.4.1, 3.4.2 and 3.4.3 for the vector basis functions $\mathrm{v}_{i}^{\tilde{f}}(p)$ with $i=1, \ldots, F$. According to such energetic approach, the number

$$
M_{i j}^{e e}(\varepsilon)=\int_{v} \mathrm{v}_{i}^{e}(p) \cdot \varepsilon \mathrm{v}_{j}^{e}(p) \mathrm{d} v
$$


is the $i, j$ entry of constitutive matrix $\mathbf{M}^{\tilde{e} e}(\varepsilon)$, the number

$$
M_{i j}^{e \tilde{f}}(\boldsymbol{\xi})=\int_{v} \mathrm{v}_{i}^{e}(p) \cdot \boldsymbol{\xi}_{j}^{\tilde{f}}(p) \mathrm{d} v
$$

is the $i, j$ entry of constitutive matrix $\mathbf{M}^{\tilde{e} \tilde{f}}(\boldsymbol{\xi})$, the number

$$
M_{i j}^{\tilde{f} e}(\boldsymbol{\zeta})=\int_{v} \mathrm{v}_{i}^{\tilde{f}}(p) \cdot \boldsymbol{\zeta} \mathrm{v}_{j}^{e}(p) \mathrm{d} v
$$

is the $i, j$ entry of constitutive matrix $\mathbf{M}^{f e}(\boldsymbol{\zeta})$, the number

$$
M_{i j}^{\tilde{f} \tilde{f}}(\boldsymbol{\mu})=\int_{v} \mathrm{v}_{i}^{\tilde{f}}(p) \cdot \boldsymbol{\mu} \mathrm{v}_{j}^{\tilde{f}}(p) \mathrm{d} v
$$

is the $i, j$ entry of constitutive matrix $\mathbf{M}^{\tilde{f} \tilde{f}}(\boldsymbol{\mu})$. It is noted that, interestingly, the integrations in (4.4)-(4.7) can be performed exactly, the vector basis function $\mathrm{v}_{i}^{e}(p), \mathrm{v}_{i}^{\tilde{f}}(p)$ being piece-wise uniform.

As it is well known [27], discrete constitutive equations over a pair of dual grids are straightforwardly achieved by combining the discrete constitutive equations over the single primal volumes $v$, rewritten in such a way that quantities $\mathbf{D}^{\tilde{e}}, \mathbf{H}^{\tilde{f}}$, computed over the dual elements $\tilde{e}_{i}, \tilde{f}_{i}$, are expressed as a function of quantities $\mathbf{E}^{e}, \mathbf{B}^{f}$, computed over the primal elements $e_{i}, f_{i}$. This expression can be easily derived, for each element $v$ separately, by rewriting (4.2) in the form

$$
\begin{aligned}
& \mathbf{D}^{\tilde{e}}=\left(\mathbf{M}^{e e}(\boldsymbol{\varepsilon})-\mathbf{M}^{e \tilde{f}}(\boldsymbol{\xi}) \mathbf{M}^{\tilde{f} \tilde{f}}(\boldsymbol{\mu})^{-1} \mathbf{M}^{\tilde{f} e}(\boldsymbol{\zeta})\right) \mathbf{E}^{e}+\mathbf{M}^{e \tilde{f}}(\boldsymbol{\xi}) \mathbf{M}^{\tilde{f} \tilde{f}}(\boldsymbol{\mu})^{-1} \mathbf{B}^{f}, \\
& \mathbf{H}^{\tilde{f}}=-\mathbf{M}^{\tilde{f} \tilde{f}}(\boldsymbol{\mu})^{-1} \mathbf{M}^{\tilde{f} e}(\boldsymbol{\zeta}) \mathbf{E}^{e}+\mathbf{M}^{\tilde{f} \tilde{f}}(\boldsymbol{\mu})^{-1} \mathbf{B}^{f} .
\end{aligned}
$$

\section{2. $\mathrm{E}, \mathrm{B}$ to $\mathrm{D}, \mathrm{H}$ constitutive relations}

In a similar way, a discrete constitutive relation can be derived by rewriting (4.1) in the alternative form

$$
\begin{aligned}
& \mathrm{D}=\left(\boldsymbol{\varepsilon}-\boldsymbol{\xi} \boldsymbol{\mu}^{-1} \boldsymbol{\zeta}\right) \mathrm{E}+\boldsymbol{\xi} \boldsymbol{\mu}^{-1} \mathrm{~B} \\
& \mathrm{H}=-\boldsymbol{\mu}^{-1} \boldsymbol{\zeta} \mathrm{E}+\boldsymbol{\mu}^{-1} \mathrm{~B},
\end{aligned}
$$

as it is always possible since the $\boldsymbol{\alpha}$ matrix is symmetric, positive definite, and by applying the energetic approach to these equations to get

$$
\begin{aligned}
& \mathbf{D}^{\tilde{e}}=\mathbf{M}^{e e}\left(\varepsilon-\boldsymbol{\xi} \boldsymbol{\mu}^{-1} \boldsymbol{\zeta}\right) \mathbf{E}^{e}+\mathbf{M}^{e f}\left(\boldsymbol{\xi} \boldsymbol{\mu}^{-1}\right) \mathbf{B}^{f}, \\
& \mathbf{H}^{\tilde{f}}=\mathbf{M}^{f e}\left(-\boldsymbol{\mu}^{-1} \boldsymbol{\zeta}\right) \mathbf{E}^{e}+\mathbf{M}^{f f}\left(\boldsymbol{\mu}^{-1}\right) \mathbf{B}^{f},
\end{aligned}
$$

in which

$$
M_{i j}^{e e}\left(\varepsilon-\boldsymbol{\xi}^{-1} \boldsymbol{\zeta}\right)=\int_{v} \mathrm{v}_{i}^{e}(p) \cdot\left(\varepsilon-\boldsymbol{\xi}^{-1} \boldsymbol{\zeta}\right) \mathrm{v}_{j}^{e}(p) \mathrm{d} v
$$

is the $i, j$ entry of constitutive matrix $\mathbf{M}^{\tilde{e} e}\left(\boldsymbol{\varepsilon}-\boldsymbol{\xi} \boldsymbol{\mu}^{-1} \boldsymbol{\zeta}\right)$, the number

$$
M_{i j}^{e f}\left(\boldsymbol{\xi} \boldsymbol{\mu}^{-1}\right)=\int_{v} \mathrm{v}_{i}^{e}(p) \cdot \boldsymbol{\xi} \boldsymbol{\mu}^{-1} \mathrm{v}_{j}^{f}(p) \mathrm{d} v
$$

is the $i, j$ entry of constitutive matrix $\mathbf{M}^{e f}\left(\boldsymbol{\xi} \boldsymbol{\mu}^{-1}\right)$, the number

$$
M_{i j}^{f e}\left(-\boldsymbol{\mu}^{-1} \boldsymbol{\zeta}\right)=-\int_{v} \mathrm{v}_{i}^{f}(p) \cdot \boldsymbol{\mu}^{-1} \boldsymbol{\zeta} \mathrm{v}_{j}^{e}(p) \mathrm{d} v
$$


is the $i, j$ entry of constitutive matrix $\mathbf{M}^{f e}(\boldsymbol{\zeta})$, the number

$$
M_{i j}^{\tilde{f} \tilde{f}}\left(\boldsymbol{\mu}^{-1}\right)=\int_{v} \mathrm{v}_{i}^{\tilde{f}}(p) \cdot \boldsymbol{\mu}^{-1} \mathrm{v}_{j}^{\tilde{f}}(p) \mathrm{d} v
$$

is the $i, j$ entry of constitutive matrix $\mathbf{M}^{f \tilde{f}}\left(\boldsymbol{\mu}^{-1}\right)$.

As it can be verified by examples, constitutive relations (4.2), (4.10) are in general different and thus provide different counterparts of constitutive relations. Also by using Properties 3.1.1, 3.1.2 and 3.1.3 for the vector basis functions $\mathrm{v}_{i}^{e}(p)$ with $i=1, \ldots, L$, and Properties 3.2.1, 3.2.2 and 3.2.3 for the vector basis functions $\mathrm{v}_{i}^{f}(p)$ with $i=1, \ldots, F$. and by rewriting (4.8), in such a way that $\mathbf{D}^{\tilde{e}}, \mathbf{B}^{f}$ are expressed as a function of $\mathbf{E}^{e}, \mathbf{H}^{\tilde{f}}$ as in (4.2), it can be proven that properties i), ii), iii) hold for the resulting discrete constitutive relations.

\section{3. $\mathrm{D}, \mathrm{B}$ to $\mathrm{E}, \mathrm{H}$ constitutive relations}

In a similar way, a different constitutive relation can be derived by rewriting (4.1) in the alternative form

$$
\begin{aligned}
& \mathrm{E}=\boldsymbol{\varepsilon}^{-1} \mathrm{D}-\boldsymbol{\xi} \boldsymbol{\mu}^{-1} \mathrm{~B}, \\
& \mathrm{H}=-\boldsymbol{\mu}^{-1} \boldsymbol{\zeta} \mathrm{E}+\left(\boldsymbol{\varepsilon}-\boldsymbol{\xi} \boldsymbol{\mu}^{-1} \boldsymbol{\zeta}\right) \mathrm{B},
\end{aligned}
$$

as it is always possible since the $\boldsymbol{\alpha}$ matrix is symmetric, positive definite, and applying the energetic approach directly to these equations to get

$$
\begin{aligned}
& \mathbf{E}^{e}=\mathbf{M}^{\tilde{e} \tilde{e}}\left(\varepsilon^{-1}\right) \mathbf{D}^{\tilde{e}}-\mathbf{M}^{\tilde{e} \tilde{f}}\left(\varepsilon^{-1} \boldsymbol{\xi}\right) \mathbf{H}^{\tilde{f}} \\
& \mathbf{B}^{f}=\mathbf{M}^{\tilde{f} \tilde{e}}\left(\boldsymbol{\zeta} \varepsilon^{-1}\right) \mathbf{D}^{\tilde{e}}+\mathbf{M}^{\tilde{f} \tilde{f}}\left(\boldsymbol{\mu}-\zeta \varepsilon^{-1} \boldsymbol{\xi}\right) \mathbf{H}^{\tilde{f}}
\end{aligned}
$$

in which

$$
M_{i j}^{\tilde{e} \tilde{e}}\left(\varepsilon^{-1}\right)=\int_{v} \mathrm{v}_{i}^{\tilde{e}}(p) \cdot \varepsilon^{-1} \mathrm{v}_{j}^{\tilde{e}}(p) \mathrm{d} v
$$

is the $i, j$ entry of constitutive matrix $\mathbf{M}^{\tilde{e} e \tilde{e}}\left(\varepsilon^{-1}\right)$, the number

$$
M_{i j}^{\tilde{e} \tilde{f}}\left(\varepsilon^{-1} \boldsymbol{\xi}\right)=\int_{v} \mathrm{v}_{i}^{\tilde{e}}(p) \cdot \boldsymbol{\varepsilon}^{-1} \boldsymbol{\xi} \mathrm{v}_{j}^{\tilde{f}}(p) \mathrm{d} v
$$

is the $i, j$ entry of constitutive matrix $\mathbf{M}^{\tilde{e} \tilde{f}}\left(\varepsilon^{-1} \boldsymbol{\xi}\right)$, the number

$$
M_{i j}^{\tilde{f} \tilde{e}}\left(\zeta \varepsilon^{-1}\right)=\int_{v} \mathrm{v}_{i}^{\tilde{f}}(p) \cdot \zeta \varepsilon^{-1} \mathrm{v}_{j}^{\tilde{e}}(p) \mathrm{d} v
$$

is the $i, j$ entry of constitutive matrix $\mathbf{M}^{\tilde{f} \tilde{e}}\left(\zeta \varepsilon^{-1}\right)$, the number

$$
M_{i j}^{\tilde{f} \tilde{f}}\left(\boldsymbol{\mu}-\boldsymbol{\zeta} \varepsilon^{-1} \boldsymbol{\xi}\right)=\int_{v} \mathrm{v}_{i}^{\tilde{f}}(p) \cdot\left(\boldsymbol{\mu}-\boldsymbol{\zeta} \varepsilon^{-1} \boldsymbol{\xi}\right) \mathrm{v}_{j}^{\tilde{f}}(p) \mathrm{d} v
$$

is the $i, j$ entry of constitutive matrix $\mathbf{M}^{f \tilde{f}}\left(\boldsymbol{\mu}-\zeta \varepsilon^{-1} \boldsymbol{\xi}\right)$.

As it can be verified by examples, constitutive relations (4.10) are in general different with respect to previous constitutive relations (4.2), (4.10). By using Properties 3.3.1, 3.3.2 and 3.3.3 for the vector basis functions $\mathrm{v}_{i}^{\tilde{e}}(p)$ with $i=1, \ldots, L$, and Properties 3.4.1, 3.4.2 and 3.4.3 for the vector basis functions $\mathrm{v}_{i}^{f}(p)$ with $i=1, \ldots, F$. and by rewriting (4.16), in such a way that $\mathbf{D}^{\tilde{e}}, \mathbf{B}^{f}$ are expressed as a function of $\mathbf{E}^{e}, \mathbf{H}^{\tilde{f}}$ as in (4.2), it can be proven that properties i), ii), iii) hold for the resulting discrete constitutive relations. Also by rewritten (4.22) in such a way that quantities $\mathbf{D}^{\tilde{e}}, \mathbf{H}^{\tilde{f}}$, are expressed as a function of quantities $\mathbf{E}^{e}, \mathbf{B}^{f}$, the discrete constitutive equations over a pair of dual grids can be straightforwardly achieved by combining the discrete constitutive equations over the single primal volumes $v$. 


\subsection{Alternative $\mathrm{D}, \mathrm{B}$ to $\mathrm{E}, \mathrm{H}$ constitutive relations}

Lastly, a different discrete constitutive relation can be derived by rewriting (4.1) in the alternative form

$$
\begin{aligned}
& \mathrm{E}=\left(\boldsymbol{\varepsilon}-\boldsymbol{\xi} \boldsymbol{\mu}^{-1} \boldsymbol{\zeta}\right)^{-1} \mathrm{D}-\boldsymbol{\varepsilon}^{-1} \boldsymbol{\xi}\left(\boldsymbol{\mu}-\boldsymbol{\varepsilon}^{-1} \boldsymbol{\zeta}\right)^{-1} \mathrm{~B}, \\
& \mathrm{H}=-\boldsymbol{\mu}^{-1} \boldsymbol{\zeta}\left(\varepsilon-\boldsymbol{\xi} \boldsymbol{\mu}^{-1} \boldsymbol{\zeta}\right)^{-1} \mathrm{D}+\left(\boldsymbol{\mu}-\boldsymbol{\zeta} \varepsilon^{-1} \boldsymbol{\xi}\right)^{-1} \mathrm{~B}
\end{aligned}
$$

as it is always possible since the $\boldsymbol{\alpha}$ matrix is symmetric, positive definite, and applying the energetic approach directly to these equations to get

$$
\begin{aligned}
& \mathbf{E}^{e}=\mathbf{M}^{\tilde{e} e}\left(\left(\varepsilon-\boldsymbol{\xi} \boldsymbol{\mu}^{-1} \boldsymbol{\zeta}\right)^{-1}\right) \mathbf{D}^{\tilde{e}}-\mathbf{M}^{\tilde{e} f}\left(\varepsilon^{-1} \boldsymbol{\xi}\left(\boldsymbol{\mu}-\boldsymbol{\varepsilon}^{-1} \boldsymbol{\zeta}\right)^{-1}\right) \mathbf{B}^{f}, \\
& \mathbf{H}^{f}=-\mathbf{M}^{f \tilde{e}}\left(\boldsymbol{\mu}^{-1} \boldsymbol{\zeta}\left(\varepsilon-\boldsymbol{\xi} \boldsymbol{\mu}^{-1} \boldsymbol{\zeta}\right)^{-1}\right) \mathbf{D}^{\tilde{e}}+\mathbf{M}^{f f}\left(\left(\boldsymbol{\mu}-\boldsymbol{\zeta} \varepsilon^{-1} \boldsymbol{\xi}\right)^{-1}\right) \mathbf{B}^{f},
\end{aligned}
$$

in which

$$
M_{i j}^{\tilde{e} \tilde{e}}\left(\left(\varepsilon-\boldsymbol{\xi} \boldsymbol{\mu}^{-1} \boldsymbol{\zeta}\right)^{-1}\right)=\int_{v} \mathrm{v}_{i}^{\tilde{e}}(p) \cdot\left(\varepsilon-\boldsymbol{\xi} \boldsymbol{\mu}^{-1} \boldsymbol{\zeta}\right)^{-1} \mathrm{v}_{j}^{\tilde{e}}(p) \mathrm{d} v
$$

is the $i, j$ entry of constitutive matrix $\mathbf{M}^{\tilde{e} \tilde{e}}\left(\left(\varepsilon-\boldsymbol{\xi} \boldsymbol{\mu}^{-1} \boldsymbol{\zeta}\right)^{-1}\right)$, the number

$$
M_{i j}^{\tilde{e} f}\left(\varepsilon^{-1} \boldsymbol{\xi}\left(\boldsymbol{\mu}-\boldsymbol{\varepsilon}^{-1} \boldsymbol{\zeta}\right)^{-1}\right)=\int_{v} \mathrm{v}_{i}^{\tilde{e}}(p) \cdot \boldsymbol{\varepsilon}^{-1} \boldsymbol{\xi}\left(\boldsymbol{\mu}-\boldsymbol{\varepsilon}^{-1} \boldsymbol{\zeta}\right)^{-1} \mathrm{v}_{j}^{f}(p) \mathrm{d} v
$$

is the $i, j$ entry of constitutive matrix $\mathbf{M}^{\tilde{e} f}\left(\varepsilon^{-1} \boldsymbol{\xi}\left(\boldsymbol{\mu}-\boldsymbol{\varepsilon}^{-1} \boldsymbol{\zeta}\right)^{-1}\right)$, the number

$$
M_{i j}^{f \tilde{e}}\left(\boldsymbol{\mu}^{-1} \boldsymbol{\zeta}\left(\varepsilon-\boldsymbol{\xi} \boldsymbol{\mu}^{-1} \boldsymbol{\zeta}\right)^{-1}\right)=\int_{v} \mathrm{v}_{i}^{f}(p) \cdot \boldsymbol{\mu}^{-1} \boldsymbol{\zeta}\left(\varepsilon-\boldsymbol{\xi} \boldsymbol{\mu}^{-1} \boldsymbol{\zeta}\right)^{-1} \mathrm{v}_{j}^{\tilde{e}}(p) \mathrm{d} v
$$

is the $i, j$ entry of constitutive matrix $\mathbf{M}^{f \tilde{e}}\left(\boldsymbol{\mu}^{-1} \boldsymbol{\zeta}\left(\boldsymbol{\varepsilon}-\boldsymbol{\xi} \boldsymbol{\mu}^{-1} \boldsymbol{\zeta}\right)^{-1}\right)$, the number

$$
M_{i j}^{f f}\left(\left(\boldsymbol{\mu}-\boldsymbol{\zeta} \varepsilon^{-1} \boldsymbol{\xi}\right)^{-1}\right)=\int_{v} \mathrm{v}_{i}^{f}(p) \cdot\left(\boldsymbol{\mu}-\boldsymbol{\zeta} \varepsilon^{-1} \boldsymbol{\xi}\right)^{-1} \mathrm{v}_{j}^{f}(p) \mathrm{d} v
$$

is the $i, j$ entry of constitutive matrix $\mathbf{M}^{f f}\left(\left(\boldsymbol{\mu}-\zeta \varepsilon^{-1} \boldsymbol{\xi}\right)^{-1}\right)$.

As it can be verified by examples, constitutive relations (4.22) are in general different with respect to previous constituive relations (4.2), (4.10), (4.16). By using Properties 3.3.1, 3.3.2 and 3.3.3 for the vector basis functions $\mathrm{v}_{i}^{\tilde{e}}(p)$ with $i=1, \ldots, L$, and Properties 3.2.1, 3.2.2 and 3.2.3 for the vector basis functions $\mathrm{v}_{i}^{f}(p)$ with $i=$ $1, \ldots, F$. and by rewriting (4.8), in such a way that $\mathbf{D}^{\tilde{e}}, \mathbf{B}^{f}$ are expressed as a function of $\mathbf{E}^{e}, \mathbf{H}^{\tilde{f}}$ as in (4.2), it can be proven that properties i), ii), iii) hold for the resulting discrete constitutive relations. Also by rewritten (4.22) in such a way that quantities $\mathbf{D}^{\tilde{e}}, \mathbf{H}^{\tilde{f}}$, are expressed as a function of quantities $\mathbf{E}^{e}$, $\mathbf{B}^{f}$, the discrete constitutive equations over a pair of dual grids can be straightforwardly achieved by combining the discrete constitutive equations over the single primal volumes $v$.

\section{Numerical Results}

The introduced constitutive matrices can be used to solve various problems arising in computational physics. Here we focus on a reference magnetostatic problem which is solved by using a pair of complementary geometric formulations. The two formulations are based on circulation on primal edges of a magnetic vector potential $A$ and on a magnetic scalar potential $\Omega$ defined in primal nodes respectively; see for example [24] for a detailed description. The magnetostatic formulations need the reluctance and the permeance constitutive matrices described in what follows. 


\subsection{Reluctance matrix using $\mathrm{v}_{i}^{f}$}

The reluctance matrix $\boldsymbol{\nu}=\mathbf{M}^{f \tilde{f}}(\nu)$ for polyhedron $v$ relates the magnetic induction flux $\Phi_{i}$ associated with $f_{i}$ with the magneto-motive forces (m.m.f.s) $F_{i}$ associated with $\tilde{f}_{i}$, with $i=1, \ldots, F$, where $\nu$ is the uniform reluctivity in $v ; \operatorname{dim}(\boldsymbol{\nu})=F$ holds. The entries of $\boldsymbol{\nu}$ are

$$
\nu_{i j}=M_{i j}^{f \tilde{f}}(\nu)=\int_{v} \mathrm{v}_{i}^{f} \cdot \nu \mathrm{v}_{j}^{f} \mathrm{~d} v .
$$

\subsection{Reluctance matrix using $\mathrm{v}_{i}^{\tilde{f}}$}

As a first step we construct the permeance matrix $\tilde{\boldsymbol{\mu}}=\mathbf{M}^{\tilde{f} f}\left(\nu^{-1}\right)$ for the polyhedron $v$ relating the m.m.f.s $F_{i}$ with the magnetic induction fluxes $\Phi_{i}$, with $i=1, \ldots, F ; \operatorname{dim}(\tilde{\boldsymbol{\mu}})=F$ holds. The entries of $\tilde{\boldsymbol{\mu}}$ are

$$
\tilde{\mu}_{i j}=M_{i j}^{\tilde{f} f}\left(\nu^{-1}\right)=\int_{v} \mathrm{v}_{i}^{\tilde{f}} \cdot \mu \mathrm{v}_{j}^{\tilde{f}} \mathrm{~d} v,
$$

where $\mu=\nu^{-1}$ is the uniform permeability in $v$. As a second step, we invert $\tilde{\boldsymbol{\mu}}$ obtaining the reluctance matrix $\tilde{\boldsymbol{\nu}}=\tilde{\boldsymbol{\mu}}^{-1}=\left(\mathbf{M}^{\tilde{f f}}\left(\nu^{-1}\right)\right)^{-1} ; \tilde{\boldsymbol{\nu}}$ is an alternative constitutive matrix with respect to $\boldsymbol{\nu}$.

\subsection{Permeance matrix using $\mathrm{v}_{i}^{e}$}

The permeance matrix $\boldsymbol{\mu}=\mathbf{M}^{e \tilde{e}}(\mu)$ for polyhedron $v$ relates the m.m.f.s $F_{i}$ associated with $e_{i}$ with the magnetic induction fluxes $\Phi_{i}$ associated with $\tilde{e}_{i}$, with $i=1, \ldots, L ; \operatorname{dim}(\boldsymbol{\mu})=L$ holds. The entries of $\boldsymbol{\mu}$ are

$$
\mu_{i j}=M_{i j}^{e \tilde{e}}(\mu)=\int_{v} \mathrm{v}_{i}^{e} \cdot \mu \mathrm{v}_{j}^{e} \mathrm{~d} v .
$$

\subsection{Permeance matrix using $\mathrm{v}_{i}^{\tilde{e}}$}

As a first step, we construct matrix $\tilde{\boldsymbol{\nu}}=\mathbf{M}^{\tilde{e} e}\left(\mu^{-1}\right)$ for polyhedron $v$ relating $\Phi_{i}$ with $F_{i} ; \operatorname{dim}(\tilde{\boldsymbol{\nu}})=L$ holds. The entries of $\tilde{\boldsymbol{\nu}}$ are

$$
\tilde{\nu}_{i j}=M_{i j}^{\tilde{e} e}\left(\mu^{-1}\right)=\int_{v} \mathrm{v}_{i}^{\tilde{e}} \cdot \nu \mathrm{v}_{j}^{\tilde{e}} \mathrm{~d} v .
$$

As a second step, we invert $\tilde{\boldsymbol{\nu}}$ obtaining the permeance matrix $\tilde{\boldsymbol{\mu}}=\left(\mathbf{M}^{\tilde{e} e}\left(\mu^{-1}\right)\right)^{-1}=\tilde{\boldsymbol{\nu}}^{-1}$. Again, $\tilde{\boldsymbol{\mu}}$ is an alternative constitutive matrix with respect to $\boldsymbol{\mu}$.

\subsection{Patch test}

To test the consistency numerically, firstly we consider a static case, where the actual fields are uniform.

In the cubical domain $D$ we constructed an Cartesian primal complex consisting of $3 \times 3 \times 3$ cubical elements, see Figure 4a. By randomly displacing some nodes, we obtain a new deformed primal complex $\mathcal{K}$ made of 27 hexahedra, see Figure 4b. Then, we apply the subgridding technique [19] by subdividing the central hexahedron in 64 hexahedra and the hexahedron below in 8 hexahedra, see Figure 4c. The final grid is formed by 97 cells, 369 faces, 478 edges, and 195 nodes. The boundary conditions are set in order to generate in $D$ a uniform magnetic induction field $\mathrm{B}$ of amplitude $1 \mathrm{~T}$ and pointing down the vertical axis. The linear systems of equations arising from the magnetostatic problem discretized by the DGA using both complementary formulations [8,24] on the polyhedral primal complex $\mathcal{K}$ are solved using the reluctance and permeance constitutive matrices described in Sections 5.1, 5.2 and 5.3, 5.4, respectively. It is possible to see in Figure 4d that the uniform field solution of the magnetostatic problem is interpolated exactly in the whole domain $D$, by using both formulations and constitutive matrices constructed by means of basis functions of the primal or dual complex.

\subsection{A magnetostatic problem}

We will now move to a non-uniform field problem. The reluctance and permeance constitutive matrices described in Sections 5.1, 5.2 and 5.3, 5.4, respectively, are used to solve a reference magnetostatic problem 


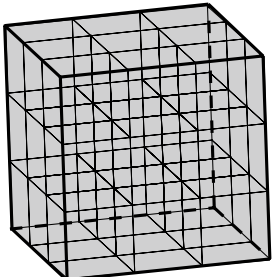

(a)

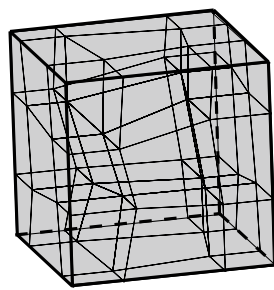

(b)

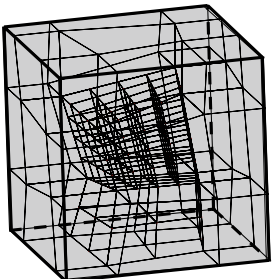

(c)

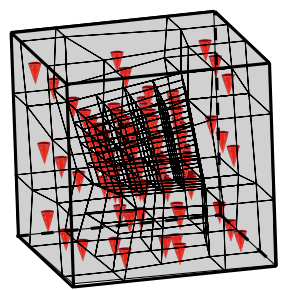

(d)

Figure 4. (a) A cube $D$ is partitioned into 27 cubes. (b) Some of the nodes are randomly displaced to obtain 27 hexahedra. (c) Two hexahedra are subdivided in 64 and 8 hexahedra, respectively, by a subgridding technique. (d) The uniform field, solution of the magnetostatic problem, is interpolated exactly in all $D$.

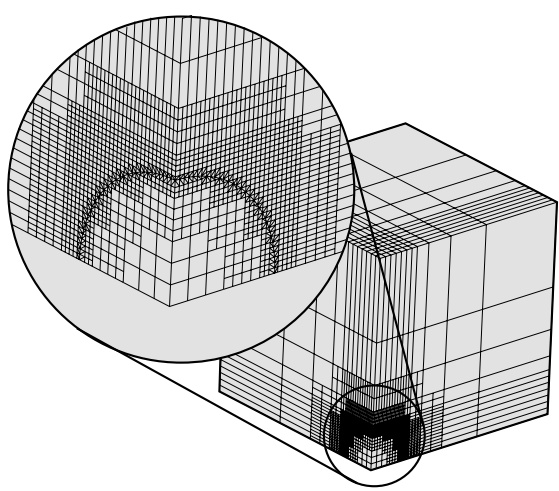

FiguRE 5 . The trace on the boundary of the domain box of the grid obtained by the subgridding of an initial hexahedral grid and successive hexahedral splitting is shown.

consisting in a sphere of radius $R=0.35 \mathrm{~m}$ made of linear magnetic medium with relative permeability $\mu_{r}=$ 1000 immersed in air. Only 1/8 of the problem is meshed with a grid made of 26121 polyhedra, 85507 faces, 88317 edges, and 28932 nodes. The primal grid is obtained by means of the subgridding of an initial coarse hexahedral grid and by cutting each hexahedra intersecting the spherical surface by means of triangles [19], as shown in Figure 5.

In Figure 6, the polyhedral grid of the sphere and the triangular faces bounding the spherical surface are represented. This kind of polyhedral elements provide a very good tessellation of the spherical surface avoiding the staircase effect.

An external uniform induction field $\mathrm{B}=B_{z} \hat{z}, B_{z}=1 \mathrm{~T}$ being the field component along the vertical axis, is enforced by boundary conditions.

The magnetostatic problem has been solved with two different formulations, which employ different kind of constitutive matrices.

The A formulation searches for a DoF-array $\mathbf{A}$ of the circulations $A$ of the magnetic vector potential along the primal edges $e$ of the mesh such that

$$
\mathbf{C}^{T} \mathbf{F}=\mathbf{I}(\mathrm{a}), \quad \mathbf{F}=\boldsymbol{\nu} \boldsymbol{\Phi}(\mathrm{b}), \quad \boldsymbol{\Phi}=\mathbf{C A}(\mathrm{c}),
$$

hold, where (5.1a) is the Ampère's Law at discrete level, $\mathbf{I}$ is the array of known solenoidal currents $I$ crossing the dual faces, $\mathbf{F}$ is the array of magneto-motive forces on dual edges, $\boldsymbol{\Phi}$ is the array of magnetic fluxes on primal 


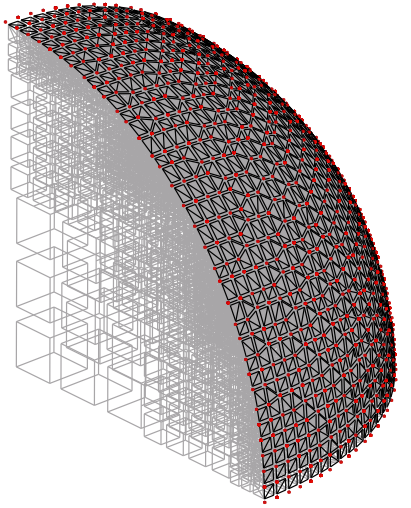

FiguRE 6. Detail of the polyhedral grid and its trace on the interface surface between sphere and air.

faces and $\mathbf{C}$ is an incidence matrix containing the incidences between each face-edge pair. Finally, (5.1c) assures that Gauss's Law at discrete level $\mathbf{D} \boldsymbol{\Phi}=\mathbf{0}$ is identically satisfied, since $\mathbf{D C}=\mathbf{0}$ holds. By substituting (5.1b) and (5.1c) in (5.1a), we obtain the algebraic system

$$
\mathbf{C}^{T} \boldsymbol{\nu} \mathbf{C A}=\mathbf{I},
$$

for which the boundary conditions must be specified in terms of $A$ on the primal edges on the boundary of $D$.

The $\Omega$ formulation searches a DoF-array $\Omega$ of magnetic scalar potentials $\Omega$ associated with the nodes $n$ such that

$$
\mathbf{G}^{T} \boldsymbol{\Phi}=0(\mathrm{a}), \quad \boldsymbol{\Phi}=\boldsymbol{\mu} \mathbf{F}(\mathrm{b}), \quad \mathbf{F}=\mathbf{G} \boldsymbol{\Omega}+\mathbf{T}(\mathrm{c})
$$

hold, where (5.3a) is Gauss' Law at discrete level, $\mathbf{G}$ is the incidence matrix that stores the incidences between each edge-node pair and $\boldsymbol{\Phi}$ is the array of magnetic fluxes on dual faces. $\mathbf{F}$ is the array of magneto-motive forces on primal edges, whereas $\mathbf{T}$ is the known array of the circulations $T$ of the electric vector potential along primal edges. The array $\mathbf{T}$ satisfies the following property $\mathbf{C} \mathbf{T}=\mathbf{I}$, where $\mathbf{I}$ is the array containing the known solenoidal currents on primal faces. $\mathbf{T}$ may be obtained by back-substitution only, i.e. without solving any global system, as indicated in [21]. Finally, (5.3c) assures that Ampère's Law at discrete level $\mathbf{C F}=\mathbf{I}$ is identically satisfied, since $\mathbf{C G}=\mathbf{0}$ holds. Then by substituting (5.3b) and (5.3c) in (5.3a), we obtain the system of equations

$$
\mathrm{G}^{T} \boldsymbol{\mu} \mathrm{G} \Omega=-\mathbf{G}^{T} \boldsymbol{\mu} \mathbf{T},
$$

where the boundary conditions must be specified in terms of $\Omega$ on the nodes on the boundary of $D$.

The magnetostatic problem using the $A$ formulation [24] consists of 88317 unknowns and it is solved in about $3.1 \mathrm{~s}$ and $5.3 \mathrm{~s}$, using the $\boldsymbol{\nu}$ and $\tilde{\boldsymbol{\nu}}$, respectively. The magnetostatic problem using the $\Omega$ formulation [24] consists of 28932 unknowns and it is solved in about $0.9 \mathrm{~s}$ and $1.8 \mathrm{~s}$, using the $\boldsymbol{\mu}$ and $\tilde{\boldsymbol{\mu}}$, respectively. Figure 7 shows the computed component $B_{z}$ along the $z$ axis of $\mathrm{B}$ using both complementary magnetostatic formulations, along a number of sample points on a horizontal line. In the same Figure, $B_{z}$ has been compared with the analytical solution showing very good agreement. Table 1 contains the numbers of iterations needed by the Conjugate Orthogonal Conjugate Gradient (COCG) method to converge at the solution of the linear systems of equations together with the error in energy norm, evaluated by using

$$
\epsilon_{B}=\sqrt{\frac{\int_{D} \nu\left|\mathrm{B}-\mathrm{B}_{r e f}\right|^{2} \mathrm{~d} V}{\int_{D} \nu\left|\mathrm{B}_{r e f}\right|^{2} \mathrm{~d} V}},
$$

which also shows a very good agreement with the analytical solution. 


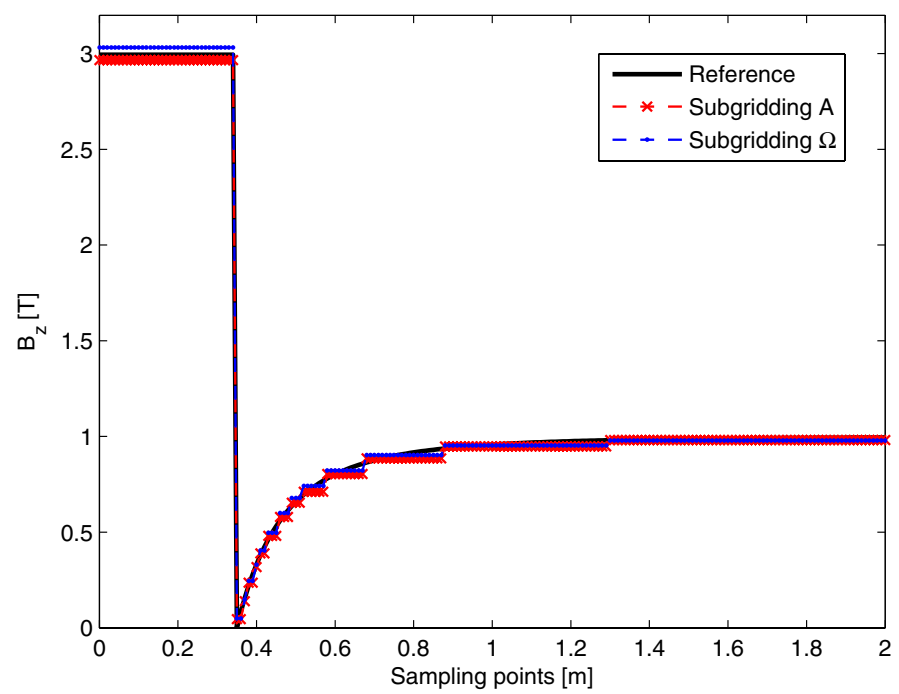

FIgURE 7 . The computed $B_{z}$ components by the complementary magnetostatic formulations on a number of sample points along a line are shown and compared with the analytical solutions.

TABLE 1. COCG iterations and errors in energy norm for each proposed constitutive matrix.

\begin{tabular}{lcccc}
\hline Constitutive matrix & $\boldsymbol{\nu}$ & $\tilde{\boldsymbol{\nu}}$ & $\boldsymbol{\mu}$ & $\tilde{\boldsymbol{\mu}}$ \\
\hline Formulation [24] & $A$ & $A$ & $\Omega$ & $\Omega$ \\
Unknowns & 88317 & 88317 & 28932 & 28932 \\
COCG iterations & 106 & 193 & 74 & 157 \\
$\epsilon_{B}[\%]$ & 0.60 & 0.86 & 0.43 & 0.78 \\
\hline
\end{tabular}

\section{Full WAVE ELECTROMAGNetics PROBlEM in BiAnisotropic MEDiUm}

A full wave electromagnetic problem in the frequency domain is considered in the waveguide $\Omega$ shown in Figure 8 . Half of the waveguide is empty and half is filled with a lossless reciprocal bianisotropic material, having tensors $\overline{\bar{\varepsilon}}$ and $\overline{\bar{\nu}}$ of vacuum and

$$
\overline{\bar{\xi}}=\overline{\bar{\zeta}}=i \kappa\left[\begin{array}{lll}
1 & 1 & 0 \\
1 & 1 & 0 \\
0 & 0 & 1
\end{array}\right]
$$

where $\kappa$ is a material dependent parameter. The fundamental Transverse Electrical mode $\left(T E_{10}\right)$ is applied at the input port, while at the output port a Perfect Electric Conductor (PEC) condition is set. The waveguide is meshed with tetrahedra defining a primal grid $\mathcal{G}$ and a barycentric dual grid $\tilde{\mathcal{G}}$ is adopted. The discretization is made by the the proposed DGA approach.

This approach searches the DoF-array $\mathbf{U}$ of the circulations of the electric field along the primal edges $e$ of the mesh such that

$$
\mathbf{C U}=-i \omega \boldsymbol{\Phi}(\mathrm{a}), \quad \boldsymbol{\Psi}=\varepsilon \mathbf{U}+\boldsymbol{\xi} \boldsymbol{\Phi}(\mathrm{b}), \quad \mathbf{F}=\boldsymbol{\zeta} \mathbf{U}+\boldsymbol{\nu} \boldsymbol{\Phi}(\mathrm{c}), \quad \mathbf{C}^{T} \mathbf{F}=i \omega \boldsymbol{\Psi}(\mathrm{d}),
$$

hold, where $\omega$ is angular frequency, (6.1a) is Faraday's Law at discrete level, $\boldsymbol{\Psi}$ is the array of electric dispacement fluxes on dual faces, $\mathbf{F}$ is the array of magneto-motive forces on dual edges, $\boldsymbol{\Phi}$ is the array of magnetic induction fluxes on primal faces and $\mathbf{C}$ is an incidence matrix containing the incidences between each face-edge pair. 


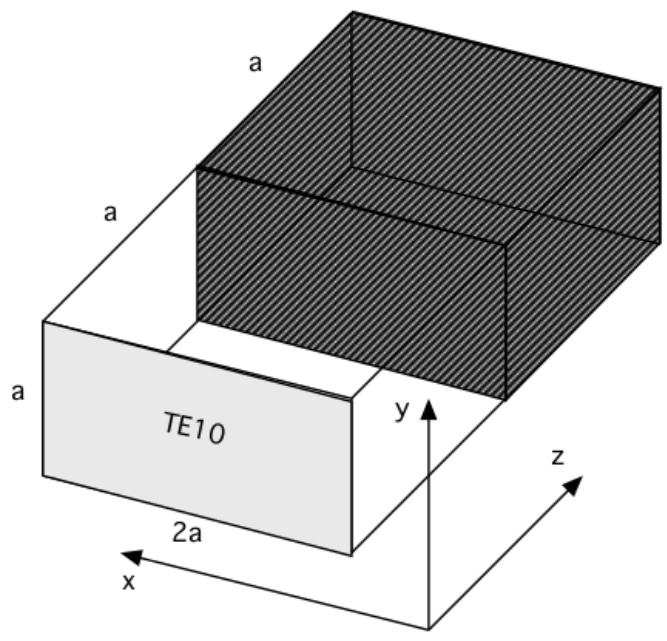

FiguRE 8. Benchmark's problem.

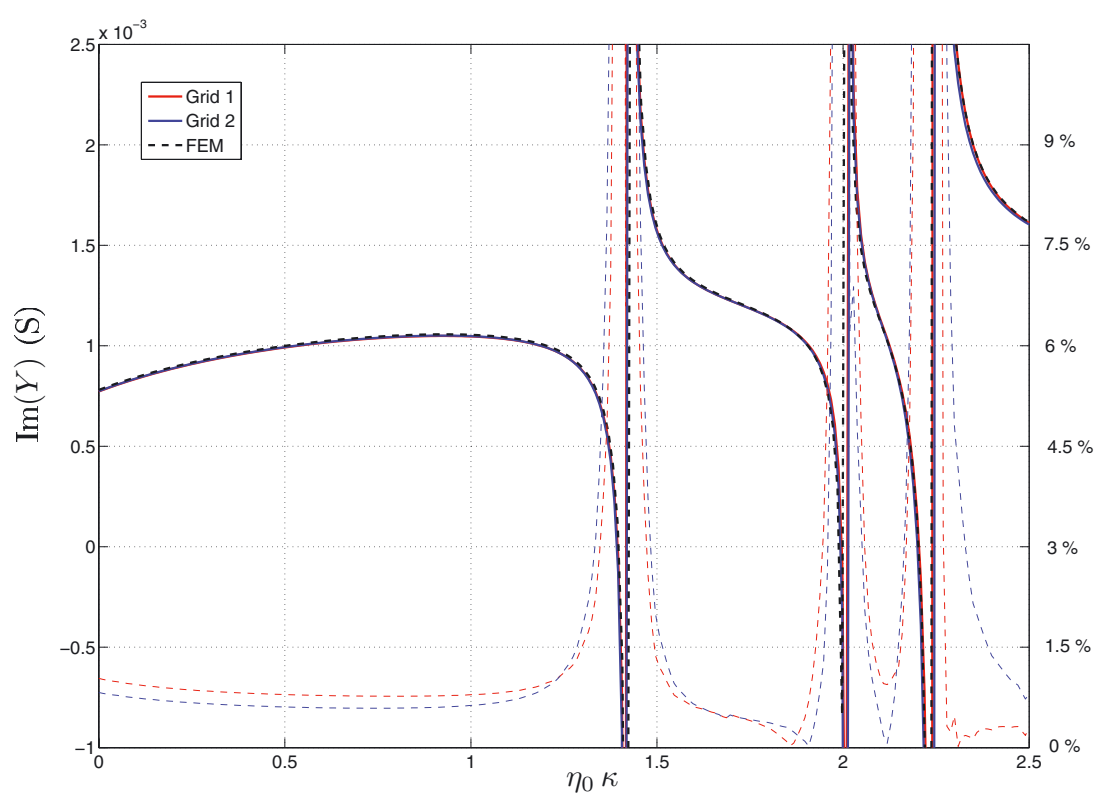

Figure 9 . Susceptance $\operatorname{Im}(Y)$ at the $T E_{10}$ port as a function of material parameter $\kappa$, for increasingly refined meshes. Percent differences with respect to the FEM solution are shown by thin dashed lines.

Finally, (6.1d) is Ampère-Maxwell's law at discrete level. By substituting (6.1b), (6.1c), (6.1d) in (6.1a), we obtain the algebraic system

$$
\left(\mathbf{C}^{T} \boldsymbol{\nu} \mathbf{C}-i \omega\left(\mathbf{C}^{T} \boldsymbol{\zeta}+\boldsymbol{\xi} \mathbf{C}\right)-\omega^{2} \varepsilon\right) \mathbf{U}=\mathbf{0}
$$

for which the boundary conditions must be specified in terms of $\mathbf{U}$ on the primal edges on the boundary of $D$.

The susceptance $\operatorname{Im}(Y(\omega))$ at the input port of the structure has been evaluated by determining the flux of Poynting's vector across it. The results for two grids with 35934 and 55456 edges, are compared in Figure 9 with an accurate FEM solution, as a function of $\kappa$. The construction and the solution of each system of equations 
over the finer grid, by the Matlab direct solver, required about $5 \mathrm{~s}$. The variation in storage requirements and computational times with respect to the case of isotropic materials is negligible.

\section{CONCLUSiOn}

New vector basis functions, which allow one to construct stable and consistent discrete constitutive equations for the Discrete Geometric Approach to computational electromagnetics even with bianisotropic media, have been introduced. The corresponding material matrices are obtained efficiently by a simple closed-form geometric construction. The results obtained by such constitutive matrices, considering both a magnetostatic and full wave electromagnetics benchmark problem, are in good agreement with the reference solutions.

\section{REFERENCES}

[1] P. Alotto and L. Codecasa, A FIT Formulation of Bianisotropic Materials Over Polyhedral Grids. IEEE Trans. Magn. 50 (2014) 7008504.

[2] L. Beirao da Veiga, A residual based error estimator for the Mimetic Finite Difference method. Numer. Math. 108 (2008) $387-406$.

[3] L. Beirao da Veiga, V. Gyrya, K. Lipnikov and G. Manzini, Mimetic finite difference method for the Stokes problem on polygonal meshes. J. Comput. Phys. 228 (2009) 7215-7232.

[4] J. Bonelle and A. Ern, Analysis of Compatible Discrete Operator schemes for elliptic problems on polyhedral meshes. ESAIM: M2AN 48 (2014) 553-581.

[5] J. Bonelle, D.A. Di Pietro and A. Ern, Low-order reconstruction operators on polyhedral meshes: Application to Compatible Discrete Operator schemes. Comput. Aid. Geom. Des. 35-36 (2015) 27-41.

[6] A. Bossavit, On the geometry of electromagnetism. 4: Maxwell's house. J. Japan Soc. Appl. Electromagn. Mech. 6 (1998) 318-326.

[7] A. Bossavit, Computational electromagnetism and geometry. 5: The Galerkin hodge. J. Japan Soc. Appl. Electromagn. Mech. 2 (2000) 203-209.

[8] A. Bossavit, Generalized Finite Differences in Computational Electromagnetics. Progress in Electromagnetics Research. Vol. 32 of PIER 32, edited by F.L. Teixeira. EMW, Cambridge, Ma (2001) 45-64.

[9] A. Bossavit and L. Kettunen, Yee-like schemes on staggered cellular Grids: A synthesis between FIT and FEM approaches. IEEE Trans. Mag. 36 (2000) 861-867.

[10] F. Brezzi and A. Buffa, Innovative mimetic discretizations for electromagnetic problems. J. Comput. Appl. Math. 234 (2010) 1980-1987.

[11] S.H. Christiansen, A construction of spaces of compatible differential forms on cellular complexes. Math. Models Methods Appl. Sci. 18 (2008) 739-757.

[12] J.C. Campbella and M.J. Shashkov, A Tensor artificial viscosity using a mimetic finite difference algorithm. J. Comput. Phys. 172 (2001) 739-765.

[13] M. Clemens and T. Weiland, Discrete Electromagnetism with the Finite Integration Technique. Vol. 32 of PIER, edited by F.L. Teixeira. EMW Publishing, Cambridge, Massachusetts, USA (2001) 65-87.

[14] L. Codecasa and F. Trevisan, Piecewise uniform bases and energetic approach for discrete constitutive matrices in electromagnetic problems. Int. J. Numer. Meth. Eng. 65 (2006) 548-565.

[15] L. Codecasa and F. Trevisan, Constitutive equations for discrete electromagnetic problems over polyhedral grids. J. Comput. Phys. 225 (2007) 1894-1918.

[16] L. Codecasa, R. Specogna and F. Trevisan, Base functions and discrete constitutive relations for staggered polyhedral grids. Comput. Methods Appl. Mech. Engrg. 198 (2009) 1117-1123.

[17] L. Codecasa, R. Specogna and F. Trevisan, Symmetric Positive-Definite Constitutive Matrices for Discrete Eddy-Current Problems. IEEE Trans. Mag. 43 (2007) 510-515.

[18] L. Codecasa, R. Specogna and F. Trevisan, Discrete constitutive equations over hexahedral grids for eddy-current problems. CMES 1 (2008) 1-14.

[19] L. Codecasa, R. Specogna and F. Trevisan, Subgridding to solving magnetostatics within Discrete Geometric Approach. IEEE Trans. Magn. 45 (2009) 1024-1027.

[20] L. Codecasa, R. Specogna and F. Trevisan, A new set of basis functions for the Discrete Geometric Approach. J. Comput. Phys. 229 (2010) 7401-7410.

[21] P. Dlotko and R. Specogna, Efficient generalized source field computation for $h$-oriented magnetostatic formulations. Eur. Phys. J. Appl. Phys. 53 (2011) 20801.

[22] T. Euler, Consistent Discretization of Maxwell's Equations on Polyhedral Grids. Ph. D. thesis, TU Darmstadt, Darmstadt, Germany (2007).

[23] R. Eymard, T. Gallouët and R. Herbin, Discretization of heterogeneous and anisotropic diffusion problems on general nonconforming meshes SUSHI: A scheme using stabilization and hybrid interfaces. IMA J. Numer. Anal. 30 (2010) 1009-1043. 
[24] F. Henrotte, R. Specogna and F. Trevisan, Reinterpretation of the nodal force method within Discrete Geometric Approaches. IEEE Trans. Magn. 44 (2008) 690-693.

[25] Y. Kuznetsov and S. Repin, Mixed Finite Element Method on Polygonal and Polyhedral Meshes. Numer. math. Adv. Appl. Springer, Berlin (2004) 615-622.

[26] I. Lindell, Methods for Electromaghnetic Field Analysis. IEEE Press, Piscataway, NJ, USA (1992).

[27] M. Marrone, Properties of Constitutive Matrices for Electrostatic and Magnetostatic Problems. IEEE Trans. Mag. 40 (2004) $1516-1520$.

[28] J.C. Nedelec, Mixed finite elements in $\mathbb{R}^{3}$. Numer. Math. 35 (1980) 315-341.

[29] J.C. Nedelec, A new family of mixed finite elements in R3. Numer. Math. 50 (1986) 57-81.

[30] T. Tarhasaari, L. Kettunen and A. Bossavit, Some realizations of a discrete Hodge operator: A reinterpretation of finite element techniques [for EM field analysis]. IEEE Trans. Magn. 35 (1999) 1494-1497.

[31] E. Tonti, Finite Formulation of the Electromagnetic Field. IEEE Trans. Mag. 38 (2002) 333-336.

[32] K. Yee, Numerical solution of initial boundary value problems involving Maxwell's equations in isotropic media. IEEE Trans. Antennas Propag. 14 (1966) 302-307. 